\title{
Immunostaining in Mohs Micrographic Surgery: A Review
}

\author{
Abdel Kader El Tal, MD, ${ }^{*}$ Ayad E. Abrou, MD ${ }^{\dagger}$ Mark A. Stiff, MD, ${ }^{* \ddagger}$ And \\ David A. MeHregan, MD*\$
}

\begin{abstract}
BACKGROUND With the advent of incorporating the immunoperoxidase staining technique into the processing of frozen tissue, the use of Mohs micrographic surgery (MMS) has been expanded to include several high-risk tumors such as lentigo maligna, malignant melanoma, and dermatofibrosarcoma protuberans.
\end{abstract}

OBJECTIVES To thoroughly review the English medical literature pertaining to the use of immunohistochemical staining techniques on frozen sections during MMS and to summarize the basic relevant outcomes from the different relevant studies.

MATERIALS AND METHODS Medline search was conducted, with the following words used in the search criteria: "Mohs surgery," "staining," "immunostaining," and "immunoperoxidase."

RESULTS Generally, all immunostains showed advantage over the traditional hematoxylin and eosin approach. Studies of MART-1 in melanoma chemosurgery indicated that it is typically crisp and has less background staining than MEL-5 and better staining consistency than HMB-45. In cases of desmoplastic melanomas, S100 is the stain of choice.

CONCLUSION Immunostaining offers an advantage in MMS. Large, randomized, prospective studies comparing the different immunostains are still lacking in the literature.

The authors have indicated no significant interest with commercial supporters.

$\mathrm{M}$ ohs micrographic surgery (MMS) is historically used for the treatment of basal cell carcinoma (BCC) and squamous cell carcinoma (SCC). It has been occasionally used for the treatment of other types of tumors such as malignant melanoma ${ }^{1}$ and microcystic adnexal carcinoma, ${ }^{2}$ as well as other malignant eccrine neoplasms, ${ }^{3}$ malignant follicular tumors, ${ }^{4}$ Merkel cell carcinoma, ${ }^{5}$ sebaceous carcinoma, ${ }^{6}$ atypical fibroxanthoma, ${ }^{7}$ malignant fibrous histiocytoma ${ }^{8}$ dermatofibrosarcoma protuberans, ${ }^{9}$ leiomyosarcoma, ${ }^{10}$ and extramammary Paget's disease. ${ }^{11}$

Immunoperoxidase technique, using formalin-fixed and paraffin-embedded tissue, normally takes several hours to process. The technique works well for regular excisions when the specimen can be processed in 24 hours but is not suitable for Mohs surgery, when tissue is regularly processed using the frozen section technique. Initially, the Mohs technique was modified to include "rush" permanent sections, particularly in instances in which frozen section reading was difficult to interpret, as in cases of lentigo maligna ${ }^{12}$ or lentigo maligna melanoma. ${ }^{13}$ Subsequently, the immunohistochemical staining technique was modified to allow for rapid staining and hence was performed on frozen tissue in Mohs surgery. ${ }^{14-16}$ One advantage of immunostaining frozen sections rather than formalin-embedded sections is the prevention of loss of antigens. In formalin-fixed tissue, the cell membrane is generally disrupted as a result of

*Dermatology Department, Wayne State University, Dearborn, Michigan; ${ }^{\dagger}$ Beacon Hill Dermatology, Dearborn, Michigan; ${ }^{\ddagger}$ Skin Cancer Center of Southeast Michigan, Southfield, Michigan; ${ }^{\S}$ Pinkus Dermatopathology Laboratory, Monroe, Michigan 
autolysis, and cell-surface antigen staining is not reliable In contrast, with frozen section staining, cytoplasmic and cell membrane antigen-staining can be displayed. ${ }^{17}$

Although previous techniques of immunostaining required immunofluorescence, necessitating the use of a specialized microscope, the current immunohistochemical staining techniques allow for examination of the slide under standard light microscopy.

\section{The Immunohistochemical Staining Technique}

The original, or direct, method of immunostaining allowed a single antibody, which is conjugated to an enzyme, to interact with an antigen present on the cell of interest. A substrate is then added, and after a reaction mediated by the conjugated enzyme, the substrate will fluoresce or form an insoluble color product deposited near the antibody (Figure 1). The pitfall of this technique is that it requires high concentrations of the antibody to obtain the staining. ${ }^{18}$ In other words, the technique has low sensitivity.

The indirect, or amplifying, technique has proven to be more efficient than the direct technique, particularly in the setting of frozen section tissue examination. In this method, the tissue is initially incubated with a primary antibody and then washed and incubated with a secondary antibody. The secondary antibody is commonly peroxidase labeled. A chro-

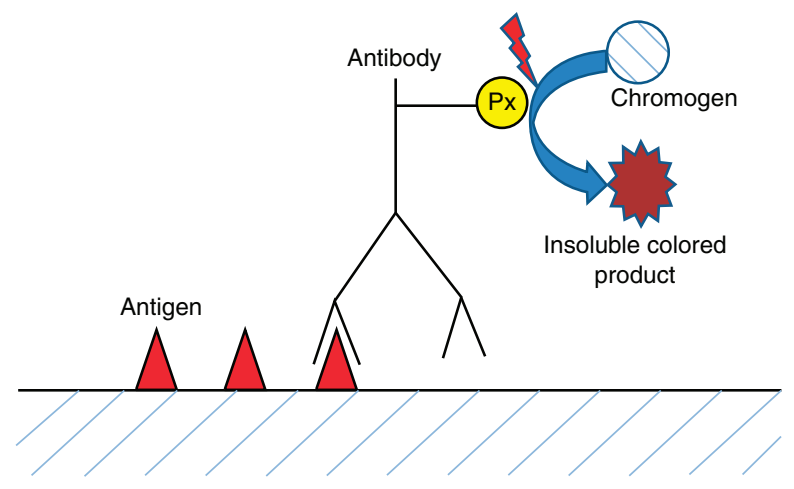

Figure 1. Direct conjugate method. The enzyme, peroxidase $(\mathrm{Px})$ in this example, is attached directly to the antibody that is specific to the antigen under study.

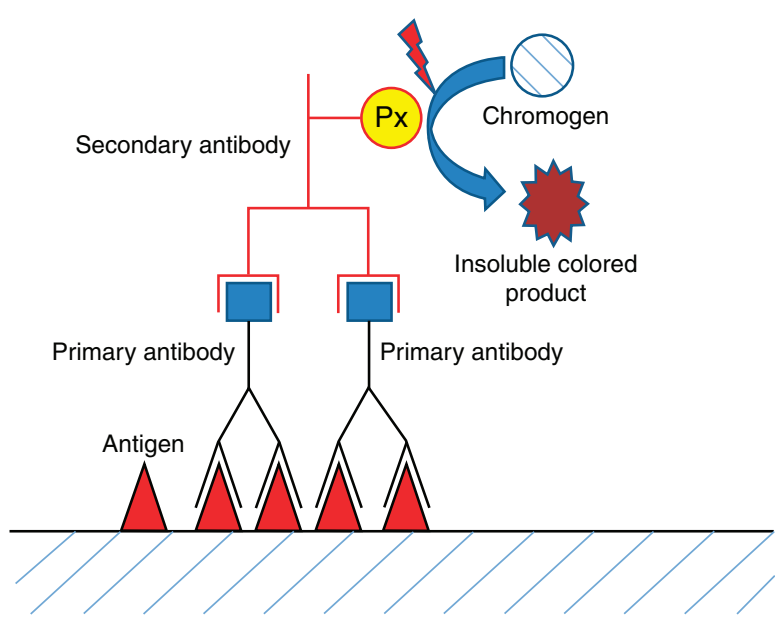

Figure 2. Indirect conjugate or sandwich method. The peroxidase $(P x)$ is attached to the secondary antibody that is specific to the primary antibody.

mogen is added afterwards and, in the presence of the peroxidase-labeled antibody, will form an insoluble colored product ${ }^{18}$ (Figure 2). Commonly used chromogens include 3 '-diaminobenzidine tetrachloride, forming a brown product, or 3-amino9 -ethylcarbazole, forming a red product. The advantage of this technique is the requirement for a lower antibody concentration to detect the antigen on formalin sections, but to shorten the time required for processing of frozen sections to 30 to 90 minutes, it is necessary to increase the concentration of the antibody. Although this enhances the sensitivity of the technique, it compromises its specificity by increasing background nonspecific staining. Hence, the use of negative controls in these instances is typically advised.

Other methods of enhancing the sensitivity of the direct and indirect techniques are antigen retrieval and microwave heating in combination with heavy metal-containing solutions or citrate buffers. ${ }^{18}$ Fixation of the tissue affects many antigens, and fixed tissue may not stain with the most sensitive techniques. These modifications allow for better staining, or greater sensitivity, but again at the expense of specificity and an increase in background staining. Again, the use of negative controls is advised. 
The steps for the indirect technique are as follows:

(1) Cut frozen section 4 to $6 \mu \mathrm{m}$ thick

(2) Fix specimen in acetone

(3) Air dry or heat

(4) Rehydrate with Tris buffered saline (TBS)

(5) Apply antibody

(6) Rinse with TBS

(7) Apply linking agent

(8) Rinse with TBS

(9) Apply labeling agent

(10) Rinse with TBS

(11) Apply chromogen

(12) Rinse, dehydrate, clear, and mount

Some authors have demonstrated an enhancement in the technique by combining some steps or shortening the length of each step.

\section{Methods of Review}

A review of the English literature pertaining to immunohistochemical staining technique use in Mohs surgery was done using a Medline search. The following words were used in the search criteria: "Mohs surgery," "staining," "immunostaining," and "immunoperoxidase." Articles relevant to the use of immunohistochemical staining for frozen sections in Mohs surgery of malignant skin cancers were retrieved (Tables 1-3).

\section{Lentigo Maligna and Malignant Melanoma}

The use of MMS for treatment of lentigo maligna and malignant melanoma has historically been somewhat controversial. These disorders can be difficult to diagnose under light microscopy, particularly when it comes to differentiating atypical melanocytic proliferation from melanoma. ${ }^{19,20}$ Upon comparing hematoxylin and eosin (H\&E)stained frozen sections to permanent sections in melanoma, Cohen and colleagues found a sensitivity of $73 \%$ and a specificity of $68 \% .{ }^{21}$ In contrast, other authors have reported that the sensitivity and specificity of H\&E-stained frozen sections for the evaluation of surgical margins of lentigo maligna are $100 \%$ and $90 \%$, respectively. ${ }^{22}$ These results can be achieved only using excellent-quality sections and experienced reviewers, yielding more than a $90 \%$ survival rate at 5 years of follow-up. ${ }^{23}$

The introduction of immunostaining has significantly decreased the controversy surrounding the use of Mohs surgery for lentigo maligna and malignant melanoma. It has greatly facilitated the detection of melanocytes and the diagnosis of melanoma on permanent sections and can be used on frozen sections as well (Table 1$){ }^{24}$

According to Gross and colleagues, ${ }^{25}$ to make MMS successful for melanoma, the following four criteria must be met: the tumor cells must be visually identifiable in the sections, the tumor must be contiguous to avoid false-negative margins, the mapping and staining component must be technically feasible, and the total tissue processing time should be short enough to allow for a staged excision and repair on the same day.

Several types of immunostains have been used to identify abnormal melanocytes.

Human melanoma, black-45 (HMB-45) is a mouse monoclonal antibody that recognizes a $30-$ to $35-$ $\mathrm{kDa}$ melanosome-associated sialated glycoprotein. ${ }^{26}$ It is present in stage I and II melanosomes of neoplastic melanocytes and stage II and III melanosomes of fetuses and infants. ${ }^{26,27}$ Staining is cytoplasmic and granular and is independent of tyrosinase activity. ${ }^{27}$ The sensitivity of HMB-45 for melanocytes in melanoma on paraffin-embedded sections has been reported to be $86 \%$ to $97 \% .^{28}$ HMB-45 staining is often negative in spindle cell melanomas, including desmoplastic and neurotropic melanomas, although newer antigen retrieval techniques have been reported to increase sensitivity to $75 \%$ in spindle cell melanomas. ${ }^{29}$ 


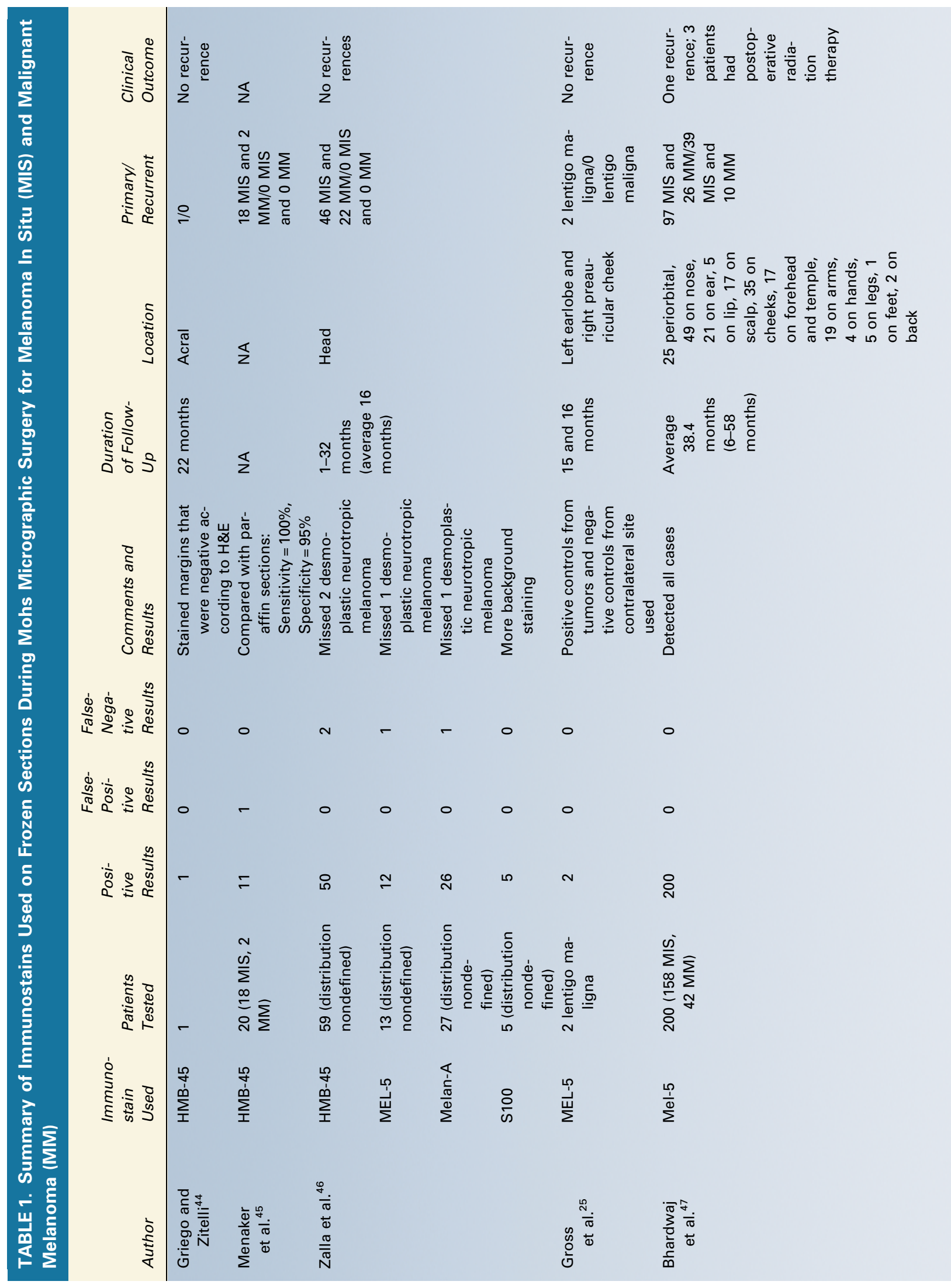




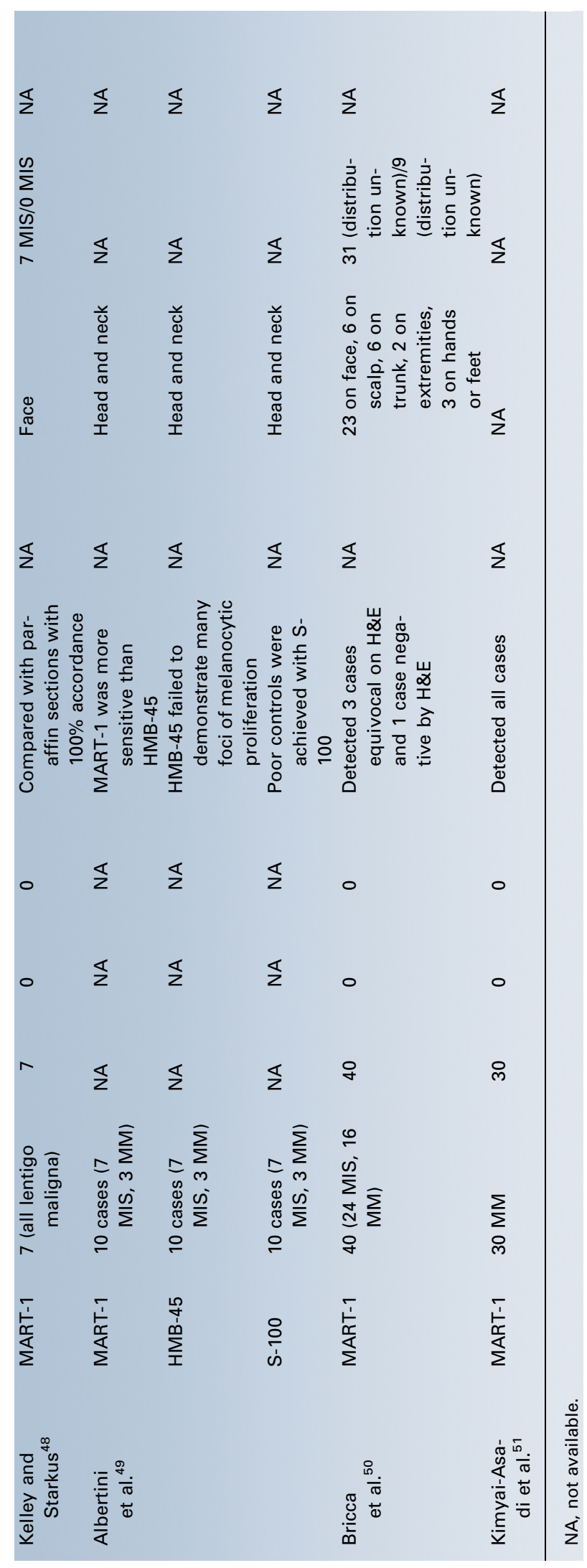

MEL-5 is a mouse monoclonal antibody that recognizes gp75, the most abundant glycoprotein in melanocytes. It is a member of the tyrosinase-related family of proteins (TRP-1) and is an integral membrane protein of melanosomes, present primarily in stage III and IV melanosomes. ${ }^{30-32}$ Also designated TA99, MEL-5 stains melanosome-containing cells, including normal epidermal melanocytes, epidermal components of benign nevi, and $95 \%$ of melanomas. However, it also stains epithelial cells in the basal layer of the epidermis through transfer of melanosomes. ${ }^{30-33}$ MEL-5 may be negative in amelanotic or desmoplastic melanoma and in the dermal component of melanoma. ${ }^{31,32}$

The Melan-A antigen, or melanoma antigen recognized by $\mathrm{T}$ cells (MART-1), is a melanocyte differentiation antigen like gp100 and gp75 (recognized by HMB-45 and MEL-5, respectively) and tyrosinase. ${ }^{34,35}$ It is a $22-\mathrm{kDa}$ cytoplasmic melanosome-associated glycoprotein recognized by murine monoclonal antibodies A-103 and M2-7C10. It is present in $80 \%$ to $100 \%$ of melanomas, resting adult melanocytes, and nevus cells in epidermal and dermal compartments. ${ }^{34-39}$ Although one study found a slightly higher sensitivity of HMB-45 for melanoma than Melan-A, ${ }^{40}$ most have noted the reverse. Blessing and colleagues ${ }^{41}$ found a $97 \%$ positivity for Melan-A and $90 \%$ positivity for HMB45 in primary melanomas. In metastatic melanomas, Melan-A stains $81 \%$ to $89 \%$ of tumors, and HMB-45 stains $75 \%$ to $76 \%$. ${ }^{35,42}$

$\mathrm{S} 100$ is a $21-\mathrm{Kd}$ protein and was originally given its name because of its solubility in $100 \%$ saturated ammonium sulfate solution. It was first found to stain human melanoma cells in 1981. Its function is nor completely understood, but it is thought to function with intracellular calcium trafficking, microtubular assay, or both. Its specificity is low because antibody to S100 stains Schwannomas, ependymomas, astrogliomas, and almost all benign and malignant melanocytic lesions and their metastases. S100 protein is also expressed in the antigen-presenting cells such as the Langerhans cells 


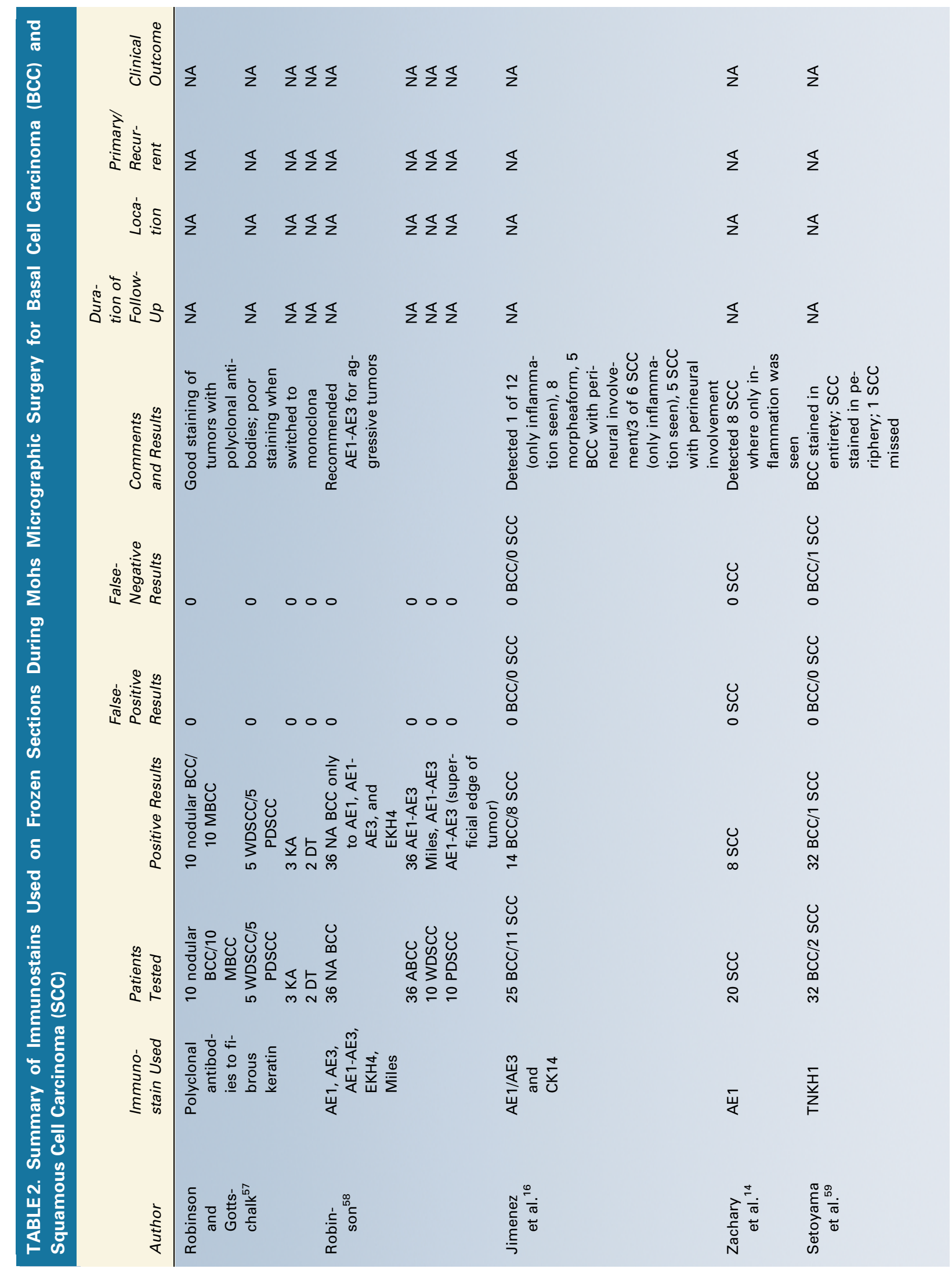




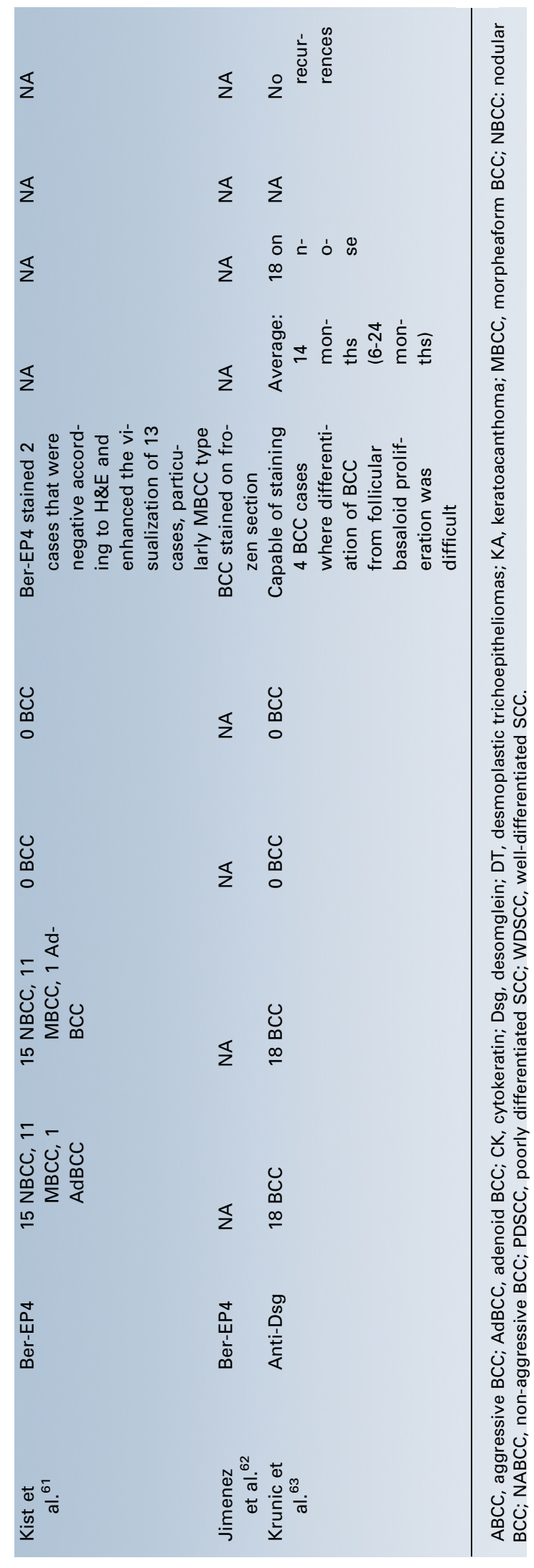

in skin and interdigitating reticulum cells in the paracortex of lymph nodes. ${ }^{43}$

Griego and Zitelli reported a case of MMS using HMB-45 for a recurrent acral melanoma. ${ }^{44}$ The authors noted that HMB-45 stained portions of the margins that were clear in frozen and permanent sections. The patient was followed up for 22 months with no recurrence seen.

Menaker and colleagues described a 90-minute protocol for HMB-45 staining on frozen section, comparing it to similar staining on permanent sections. ${ }^{45}$ Twenty patients underwent MMS with HMB-45 staining. Eleven patients were positive. One patient had a false positive result on the HMB-45. Accordingly, the HMB-45 performance on frozen sections, in comparison with permanent sections, had a sensitivity of $100 \%$ and a specificity of $95 \%$. The authors acknowledge that the specificity was decreased because of staining of non-malignant melanocytic neoplasms.

Zalla and colleagues described a 90-minute protocol for HMB-45, MEL-5, Melan-A, and S100 stains. ${ }^{46}$ They performed immunostaining on 68 tumors (46 melanomas in situ and 22 invasive melanomas). In their study, sections were stained with H\&E together with one or more immunostains to compare stain quality and results. HMB-45, MEL-5, and Melan-A all exhibited areas of crisp positive staining in areas involved by tumor. When equivocal areas were noted with one immunostain, another immunostain was performed, or the area was considered positive, and further layers were taken. In this study, HMB-45 was positive in 50 of 59 tumors $(85 \%)$. It was negative in two desmoplastic neurotropic melanomas. The two tumors were detected using immunostaining and not on H\&E-stained frozen sections. MEL-5 was performed on 13 cases and stained positive 12 of the 13 tumors (92\% of cases), including six of seven HMB-45-negative tumors. One desmoplastic neurotropic melanoma stained negative with MEL-5. MEL-5 was found to be better than S100 in intensity and specificity, with less background 


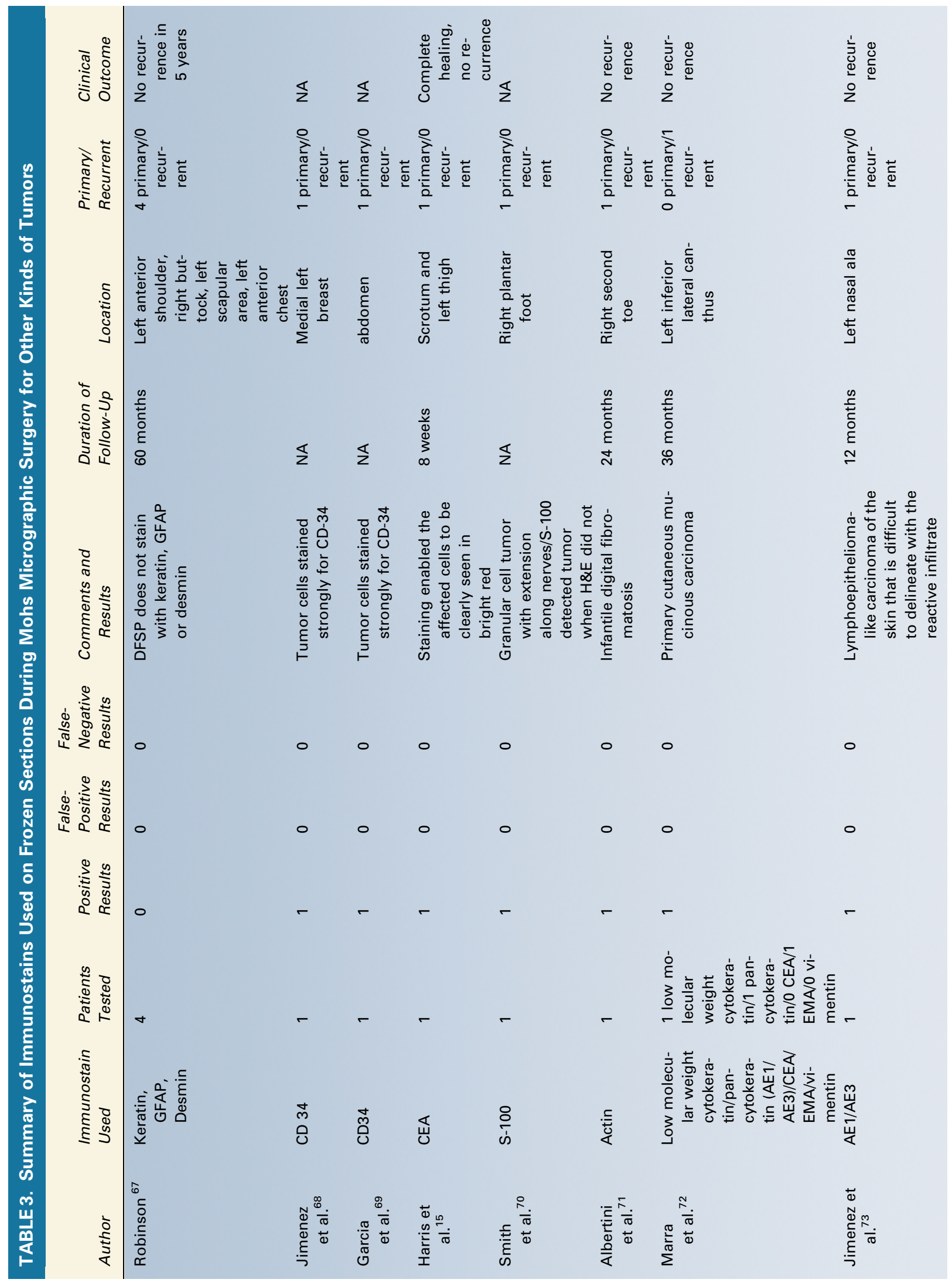




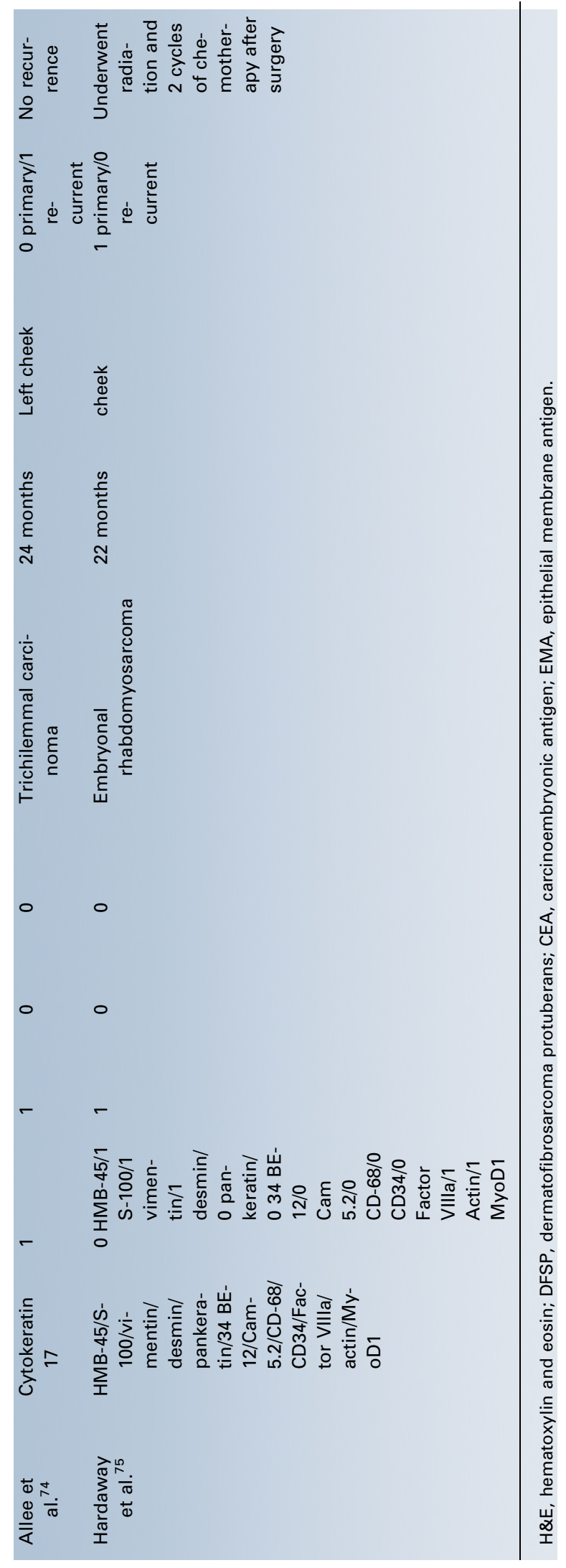

staining, although the authors concur with another study, in which it was found to be less specific than HMB-45, staining non-melanocytic lesions such as pigmented actinic keratoses, pigmented Bowen's disease, and lichen planus-like keratoses. ${ }^{32}$ Melan-A was performed on 27 cases, 26 of which were positive, including one case that was negative according to HMB-45. One desmoplastic neurotropic melanoma was weakly positive, and another was negative with this stain.

Gross and colleagues performed MMS on two cases of lentigo maligna using MEL-5. ${ }^{25}$ A 75 -minute protocol was followed. Positive controls were taken from the center of the tumors, and negative controls were taken from the contralateral sites. In the authors' opinion, MEL-5 was superior to the other immunohistochemical stains because it reliably stained epidermal melanocytes and melanoma cells. Follow-ups of 15 and 16 months revealed no recurrence of tumor.

Bhardwaj and colleagues ${ }^{47}$ studied the use of MEL-5 on 200 cases of primary or recurrent lentigo maligna and malignant melanoma. MEL-5 was done in parallel with H\&E staining. The MEL-5 staining technique required approximately 40 minutes, but with the use of an autostainer, the time needed was shortened to 20 minutes. There was no mention of MEL-5 highlighting areas not previously highlighted with H\&E staining. Of the 200 patients, three needed postoperative radiation therapy. Only one recurrence has been observed. The mean duration of follow-up in this study was 38.4 months. there was no mention of the sensitivity or the specificity of the 200 tumors studied although the authors mention a previous retrospective study that used MMS for the treatment of melanoma in situ. In that study, the sensitivity and specificity were $59 \%$ and $81 \%$, respectively, when comparing Mel-5-stained frozen sections with Mel-5-stained paraffin sections.

In 2002, Kelley and Starkus reported a series of seven patients with lentigo maligna on whom MMS was performed, and frozen sections stained with 
H\&E and MART-1 were compared with permanent sections. ${ }^{48}$ MART-1 staining on frozen sections correlated $100 \%$ with MART-1 on permanent sections and detected the atypical melanocytes in all seven cases. In this study, the authors emphasized the difficulty in discerning atypical melanocytes of lentigo maligna from the surrounding atypical melanocytic hyperplasia of sun exposure. For that purpose, sections from nine cases of normal photodamaged skin and five photoprotected cases were evaluated and used as a baseline for comparison. The authors concluded that, when the margin of lentigo maligna is evaluated, confluence of atypical melanocytes alone is not enough to label margins as positive. At minimum, there must be crowding of the atypical melanocytes.

In the same year, Albertini and colleagues reported their experience comparing MART-1, HMB-45, and S100. ${ }^{49}$ Their protocol required approximately 2 hours for the immunostaining to be completed. Some patients needed more than 1 day to achieve negative margins. Ten cases were stained with H\&E and the three mentioned stains. Positive and negative control stains were processed for each immunostain for each layer. MART-1 had better sensitivity than HMB-45 and S100 and was the preferred stain in the final pathologic determination. HMB-45 failed to demonstrate many foci of melanocytic proliferation visible with MART-1. S100 had poor controls on frozen sections, although good controls were achieved when permanent fixation was used.

In 2004, Bricca and colleagues suggested a shorter protocol for Melan-A staining. ${ }^{50}$ In brief, the protocol decreased the duration from a 90-minute to a 60 -minute protocol. In comparison with other protocols, the 1-hour protocol eliminated the linking step and shortened the protein blocking step. The protein blocking step was reduced by using a blocking agent that has high quantities of nonspecific immunoglobulin (Ig)G. The linking step was omitted by using a special secondary antibody that is bound to a spherical polymer that is directly attached to horseradish peroxidase (HRP). In this study, 40 patients with lentigo maligna and malignant melanoma were evaluated. The MART-1 stain delineated the malignant cells in three equivocal cases and one negative case according to H\&E staining.

In 2008, Kimyai-Asadi and colleagues ${ }^{51}$ reported a protocol for rapid MART-1 staining that needed only 20 minutes to perform. The heating period, fixation phase, drying phase, rehydration phase, blocking phase, antibody application phase, buffering phases, HRP application phase, and mounting phase were shorter in the 20-minute protocol than in the 1-hour protocol by Bricca and colleagues. MART-1 was able to delineate all 30 malignant melanomas studied, although there was no followup on the patients. The authors mention that MART1 enhances the sensitivity and specificity of melanoma detection on frozen section, although it is not a reliable stain for spindle cell melanoma.

Melan-A epidermal staining on frozen section is intense and crisp, with regular staining of normal basilar cells and less background staining than MEL-5, thus giving less equivocal results than MEL-5. The consistency of Melan-A stain is better than that of HMB-45, and more cases are detected than with HMB-45. ${ }^{46,49}$ However, in the case of desmoplastic melanomas, S100 offers advantages over the other stains.

\section{BCC and SCC}

Although the histologic recognition of BCC and SCC in frozen sections is straightforward in most cases, situations exist in which clear delineation and mapping of the tumor infiltration can be difficult. For example, the presence of a dense inflammatory lymphocytic infiltrate in tissue sections may make it difficult, if not impossible, to detect the malignant cells.

Cytokeratins are one of the five types of intermediate filaments that constitute the cytoskeleton of the cell. At least 20 types of cytokeratins have been characterized. Glandular epithelium is composed mainly of 
low- (LMW) to intermediate-molecular-weight keratins, and squamous epithelium consists primarily of the more complex high-molecular-weight (HMW) keratins. BCCs express cytokeratins 5, 14, 15 , and $17,,^{52-55}$ and cutaneous SCCs express cytokeratins $5,6,8,14,17$, and $18 .{ }^{53,56}$ In routine practice, a cocktail that recognizes a wide spectrum of keratins is used. AE1 detects the HMW cyto-

keratins $10,14,15$, and 16, and also the LMW cytokeratin 19 (acidic keratin). AE3 detects the HMW cytokeratins 1, 2, 3, 4, 5, and 6 and the LMW cytokeratins 7 and 8 (basic keratin). By combining these two reagents, a single reagent with a broad spectrum of reactivity against HMW and LMW cytokeratins is obtained.

In 1984, Robinson and Gottschalk ${ }^{57}$ evaluated several tumors using cytokeratin antibodies using two kinds of techniques: immunofluorescence and immunoperoxidase (Table 2). Their study indicated that immunoperoxidase is sensitive in recognizing BCCs, keratoacanthomas, and desmoplastic trichoepitheliomas. Monoclonal and polyclonal antibodies were used. Although polyclonal antikeratin antibodies stained all tumors mentioned previously, the intensity of the staining correlated with the degree of differentiation of the tumor. Monoclonal antibodies, on the other hand, stained BCC cells with varying intensity and did not stain poorly differentiated SCC.

In 1987, Robinson used several monoclonal antikeratin antibody immunostains to compare invasive BCC and SCC with their less aggressive counterparts. ${ }^{58}$ AE1, AE3, EKH4, Miles, and AE1-AE3 combination antibodies were used. EKH4 is a monoclonal mouse antibody derived from human trichilemmoma cells. Miles antibodies are derived from bovine hoof prekeratin antibodies and will stain the granular and corneal epidermal layers. Nodular BCC exhibited staining with AE1, EKH4, and an AE1-AE3 combination but not Miles antibodies. As the tumor becomes more aggressive, AE1AE3 still stain the tumor, but EKH4 and AE1 will stain only peripheral cells and ultimately becomes occasional and nonreliable. Well-differentiated SCC displayed strong staining for the Miles antibody in keratin pearls and AE1-AE3 staining throughout the tumor. In contrast to nodular BCC, AE1 and EKH4 only weakly and occasionally stained the malignant cells. As the SCC became more invasive, there was loss of the AE1-AE3 stain at the deeper margins.

In 1994, Zachary and colleagues used cytokeratin AE1 in 20 cases of SCC. ${ }^{14}$ The immunostain detected all 20 SCCs. In eight of 20 cases, small clumps or single cells of residual tumor were identified using cytokeratin-positive staining. The residual tumor often resided in areas of inflammation. Positive controls were taken from the vertical sections of the primary tumor. In the papillary dermis, the occasional presence of globular material that stained positive with the procedure was noted and might have beeen mistaken for a false-positive result if no positive controls had been used.

Jimenez and colleagues ${ }^{16}$ used a broad-spectrum anticytokeratin (AE1/AE3) together with anticytokeratin 14 in the detection of BCC and SCC during MMS. They described a 1-hour protocol for the anticytokeratin stain. In this study, the immunostaining technique was able to pick up one of 12 BCCs, and three of six SCCs where only dense inflammatory infiltrate was seen on H\&E. The stain was also able to delineate and map out more accurately subtle tumor islands in eight cases of morpheaform BCCs and highlighted perineural involvement in five cases of BCC and five cases of SCC with perineural involvement.

Immunostains other than cytokeratins have also been used. TNKH1, an antiglycoprotein antibody, is a monoclonal antibody that was primarily developed in mice to recognize differentiation antigen of a human melanoma cell line (A375). It was also found to stain epithelial tumors considered to be derived from or differentiating toward hair follicle. In their study, Setoyama and colleagues ${ }^{59}$ showed that TNKH1 stained 32 of 32 BCCs, labeling the tumors in their entirety. The antibody was useful in delineating the 
upper hair follicle and eccrine duct from BCC tumors. The antibody also irregularly stained one of two SCCs over the periphery of the tumor.

Ber-EP4 is an antibody raised against an epitope of the protein region of two human epithelial membrane glycoproteins. ${ }^{60}$ Its ability to differentiate BCC from SCC has been demonstrated previously. Kist and colleagues ${ }^{61}$ and Jimenez and colleagues ${ }^{62}$ found BerEP4 to be useful during MMS for BCC tumors.

Krunic and colleagues used the monoclonal antibody 33-3D, a mouse IgM monoclonal antibody that recognizes the cytoplasmic domains of human desmoglein, during MMS for 18 cases of BCC. ${ }^{63}$ The protocol described for staining required approximately 60 minutes to perform. The antibody shows intense pericellular staining around normal cells and will show general or diffuse cytoplasmic staining or a reduction in staining in areas of tumors. The antibody helps to differentiate BCC from hair follicles or from folliculocentric basaloid proliferation (FBP) in horizontal sections. ${ }^{63} \mathrm{FBP}$ is vertically oriented basaloid proliferations that can radiate outward and downward from the hair follicle and up toward the skin edges. It surrounds BCC and may extend inward to the proliferating border of the neoplasm. ${ }^{64} \mathrm{In}$ another study, Krunic and colleagues stained 24 SCCs and 12 keratoacanthomas (KAs) using the antidesmoglein antibodies on frozen sections from excised lesions. ${ }^{65}$ All 12 KAs showed extensive, uniform pericellular staining for desmoglein throughout the nonkeratinized layers of the tumor, whereas all five SCCs showed only focal pericellular staining, and seven SCCs showed absence of staining.

\section{Other Kinds of Tumors}

\section{Dermatofibrosarcoma Protuberans}

Dermatofibrosarcoma protuberans (DFSP) is a tumor that is commonly removed using MMS. The problem is that its histologic differentiation on H\&E-stained frozen sections can be difficult, par- ticularly at the margin of the tumor and at areas of scarring from previous surgical procedures. Traditional surgical excision usually fails to treat the microscopic extensions, and in most series, recurrence rates of $49 \%$ to $53 \%$ have been reported, with the majority developing 1 to 2 years after therapy. ${ }^{66}$ When surgical margins of $3 \mathrm{~cm}$ have been used, the recurrence rate drops to $10 \%$ to $20 \%{ }^{8}$

In 1985, Robinson reported a 5-year prospective study of four cases of DFSP treated using MMS. ${ }^{67}$ Staining with keratin, glial fibrillary acid protein, and desmin were attempted but were negative in all four tumors (Table 3).

CD34 antigen is typically found in hematopoietic stem cells, endothelium, dermal and periadnexal dendritic cells, and endoneuronal dendritic cells. Its detection helps in differentiating DFSP from keloids, dermatofibroma, atypical fibroxanthoma, and malignant fibrous histiocytoma. There is greater variability of CD34 staining in nodular areas than in plaque areas of DFSP. ${ }^{67}$

In 1994, Jimenez and colleagues used CD-34 immunostaining on MMS for a DFSP over the left medial breast of a 13-year-old girl. ${ }^{68}$ The protocol described took approximately 1 hour. The tumor immunostaining was strongly positive. Positive control was used from the first stage, where tumor was present.

In 1996, Garcia and colleagues reported the use of CD-34 immunostaining during MMS on another patient with a DFSP over the abdomen. ${ }^{69}$ The tumor again strongly stained with CD-34, although it was negative on the initial biopsy. The authors recommend biopsies from the plaque areas of the tumor with the inclusion of fatty tissue to improve the diagnostic yield of the CD-34 immunostain.

\section{Miscellaneous Tumors}

Harris and colleagues reported the treatment of extramammary Paget's disease on one patient using 
carcinoembryonic antigen (CEA) during MMS. ${ }^{15}$ The use of a quick-staining immunoalkaline phosphatase kit that, when combined with high-affinity primary antibodies, reduced the total staining time to 30 minutes, producing moderate-intensity cytoplasmic staining. As an internal control, the CEA stains eccrine and apocrine cells uniformly throughout the cytoplasm. Although Periodic Acid-Schiff stain can be used during MMS for extramammary Paget's disease, the authors find CEA to be a superior diagnostic method.

Smith and colleagues reported the treatment of one case of granular cell tumor on the right plantar foot. ${ }^{70}$ In their case report, the tumor was tracking along the nerves, and the last three layers during the surgery showed positivity only with S100 but not with the H\&E staining.

Albertini and colleagues have used actin stain in addition to H\&E and Masson trichrome in a case of a tender infantile digital fibromatosis. ${ }^{71}$ Although the tumor involved the joint capsule and was left positive at that margin, 2-year follow-up showed no recurrence of disease.

Marra and colleagues described a 45- to 60-minute protocol using automated staining for LMW cytokeratin, pancytokeratin (AE1/AE3), CEA, epithelial membrane antigen (EMA), and vimentin during MMS for one case of primary cutaneous mucinous carcinoma. ${ }^{72}$ In this report, the tumor was positive for LMW cytokeratin, AE1/AE3, and less clearly EMA but was negative for CEA and vimentin. The authors commented that the adnexal structures normally stained positive with the LMW cytokeratin and could thereby confound the interpretation of immunostained slides, whereas the tumor cells lacked histopathologic characteristics of acinar or ductal elements.

Jimenez and colleagues used pan-cytokeratin staining (AE1/AE3) during MMS for lymphoepitheliomalike carcinoma of the skin. ${ }^{73}$ The staining was particularly helpful in some areas where the tumor cells were difficult to delineate within the reactive infiltrate. Normal epidermis was used as positive internal control, and negative control runs were performed in parallel. The patient was free of recurrence after a follow-up of 12 months.

Allee and colleagues have used cytokeratin-17 during MMS of a recurrent trichilemmal carcinoma over the left cheek. ${ }^{74}$ Positive and negative margins were taken. The tumor showed strong cytoplasmic staining of the tumor cells for cytokeratin-17 and failed to stain for cytokeratin-15. Cytokeratin-17 is an intermediate filament expressed constitutively in the outer root sheath of the hair follicles but not in the interfollicular epidermis, whereas cytokeratin-15 is expressed in a subpopulation of keratinocytes in the bulge area of the outer root sheath.

Hardaway and colleagues ${ }^{75}$ have used HMB-45, S100, vimentin, desmin, pankeratin, 34 BE-12, Cam5.2, CD-68, CD34, Factor VIIIa, actin, and MyoD1 during MMS for an embryonal rhabdomyosarcoma of the cheek. S100, vimentin, desmin, actin and MyoD1 were the only positive stains. The authors emphasize that MyoD1 and myogenin are DNA-binding proteins that are involved in the differentiation of mesenchymal progenitor cells and have been used as markers in characterizing rhabdomyosarcoma.

\section{Prevalence and Costs}

In 2001, Robinson conducted a survey of 108 Mohs surgery laboratories. ${ }^{76}$ Thirteen laboratories were doing immunostaining at the time $(13 \%)$. Performance of immunostaining was associated with resections of melanoma or DFSP, with only one laboratroy using an automated immunostainer. HMB- 45 was used by $50 \%$ of the labs, S100 by $42 \%$, Mart- 1 and MEL- 5 by $42 \%$, antikeratin by $42 \%$, and anti-CD34 by $33 \%$. The author emphasized that, by using polyclonal antibodies instead of monoclonal antibodies and by using higher antibody titers, the processing time would be further shortened and would fall within the expectations of Mohs 
surgeons. The International Classification of Diseases, Ninth Revision, code for immunostaining sections in Mohs surgery is 88,342, with a current Medicare reimbursement of approximately $\$ 100$. The current estimate of cost per slide is between $\$ 20$ and $\$ 25$.

\section{Conclusion}

The use of immunohistochemical staining during MMS is definitely on the rise, particularly in cases of lentigo maligna and malignant melanoma, but large randomized prospective studies comparing the different immunostains are lacking in the literature. As a result of increasing familiarity with the stains, faster processing, and lowering costs of the antibodies, Mohs surgeons should be encouraged to integrate immunostaining into their laboratory routine.

\section{References}

1. Mohs FE. Chemosurgery for melanoma. Arch Dermatol 1977;113:285-91.

2. Leibovitch I, Huilgol SC, Selva D, et al. Microcystic adnexal carcinoma: treatment with Mohs micrographic surgery. J Am Acad Dermatol 2005;52:295-300.

3. Wildemore JK, Lee JB, Humphreys TR. Mohs surgery for malignant eccrine neoplasms. Dermatol Surg 2004;30(12 Pt 2):1574-9.

4. Garrett AB, Azmi FH, Ogburia KS. Trichilemmal carcinoma: a rare cutaneous malignancy: a report of two cases. Dermatol Surg 2004;30:113-5.

5. Boyer JD, Zitelli JA, Brodland DG, D’Angelo G. Local control of primary Merkel cell carcinoma: review of 45 cases treated with Mohs micrographic surgery with and without adjuvant radiation. J Am Acad Dermatol 2002;47:885-92.

6. Snow SN, Larson PO, Lucarelli MJ, et al. Sebaceous carcinoma of the eyelids treated by Mohs micrographic surgery: report of nine cases with review of the literature. Dermatol Surg 2002;28: 623-31.

7. Seavolt M, McCall M. Atypical fibroxanthoma: review of the literature and summary of 13 patients treated with Mohs micrographic surgery. Dermatol Surg 2006;32:435-41.

8. Brown MD, Swanson NA. Treatment of malignant fibrous histiocytoma and atypical fibrous xanthomas with micrographic surgery. J Dermatol Surg Oncol 1989;15:1287-92.

9. Nelson RA, Arlette JP. Mohs micrographic surgery and dermatofibrosarcoma protuberans: a multidisciplinary approach in 44 patients. Ann Plast Surg 2008;60:667-72.
10. Huether MJ, Zitelli JA, Brodland DG. Mohs micrographic surgery for the treatment of spindle cell tumors of the skin. J Am Acad Dermatol 2001;44:656-9.

11. Hendi A, Brodland DG, Zitelli JA. Extramammary Paget's disease: surgical treatment with Mohs micrographic surgery. J Am Acad Dermatol 2004;51:767-73.

12. Dhawan SS, Wolf DJ, Rabinovitz HS, Poulos E. Lentigo maligna. The use of rush permanent sections in therapy. Arch Dermatol 1990;126:928-30.

13. Stonecipher MR, Leshin B, Patrick J, White WL. Management of lentigo maligna and lentigo maligna melanoma with paraffinembedded tangential sections: utility of immunoperoxidase staining and supplemental vertical sections. J Am Acad Dermatol 1993;29:589-94.

14. Zachary CB, Rest EB, Furlong SM, et al. Rapid cytokeratin stains enhance the sensitivity of Mohs micrographic surgery for squamous cell carcinoma. J Dermatol Surg Oncol 1994;20:530-5.

15. Harris DW, Kist DA, Bloom K, Zachary CB. Rapid staining with carcinoembryonic antigen aids limited excision of extramammary Paget's disease treated by Mohs surgery. J Dermatol Surg Oncol 1994;20:260-4.

16. Jimenez FJ, Grichnik JM, Buchanan MD, Clark RE. Immunohistochemical techniques in Mohs micrographic surgery: their potential use in the detection of neoplastic cells masked by inflammation. J Am Acad Dermatol 1995;32:89-94.

17. Robinson JK. A review of immunohistochemical techniques for cutaneous carcinoma. Adv Dermatol 1987;2:187-205.

18. Mondragon RM, Barrett TL. Current concepts: the use of immunoperoxidase techniques in mohs micrographic surgery. J Am Acad Dermatol 2000;43(1 Pt 1):66-71.

19. Shafir R, Hiss J, Tsur H, Bubis JJ. Pitfalls in frozen section diagnosis of malignant melanoma. Cancer 1983;51:1168-70.

20. Little JH, Davis NC. Frozen section diagnosis of suspected malignant melanoma of the skin. Cancer 1974;34:1163-72.

21. Cohen LM, McCall MW, Hodge SJ, et al. Successful treatment of lentigo maligna and lentigo maligna melanoma with Mohs' micrographic surgery aided by rush permanent sections. Cancer 1994;73:2964-70.

22. Zitelli JA, Moy RL, Abell E. The reliability of frozen sections in the evaluation of surgical margins for melanoma. J Am Acad Dermatol 1991;24:102-6.

23. Zitelli JA, Brown C, Hanusa BH. Mohs micrographic surgery for the treatment of primary cutaneous melanoma. J Am Acad Dermatol 1997;37(2 Pt 1):236-45.

24. Ruiter DJ, Bröcker EB. Immunohistochemistry in the evaluation of melanocytic tumors. Semin Diagn Pathol 1993;10:76-91.

25. Gross EA, Andersen WK, Rogers GS. Mohs micrographic excision of lentigo maligna using Mel-5 for margin control. Arch Dermatol 1999;135:15-7.

26. Chiamenti AM, Vella F, Bonetti F, et al. Anti-melanoma monoclonal antibody HMB-45 recognizes a 30-35 kDa melanosomeassociated sialated glycoprotein. Melanoma Res 1996;6:291-8.

27. Kikuchi A, Shimizn H, Nishikawa T. Expression and ultrastructural localization of HMB-45 antigen. Br J Dermatol 1996;135:400-5. 
28. Zalla MJ, Lim KK, Dicaudo DJ, Gagnot MM. Mohs micrographic excision of melanoma using immunostains. Dermatol Surg 2000;26:771-84.

29. Anstey A, Cerio R, Ramnarian N, et al. Desmoplastic malignant melanoma: an immunohistochemical study of 25 cases. Am J Dermatopathol 1994;16:14-22.

30. Vijayasaradhi S, Houghton AN. Purification of an autoantigenic $75 \mathrm{kDa}$ human melanosomal glycoprotein. Int J Cancer 1991;47:298-303.

31. Thomson TM, Real FX, Murakami S, et al. Differentiation antigens of melanocytes and melanoma: analysis of melanosome and cell surface markers of human pigmented cells with monoclonal antibodies. J Invest Dermatol 1988;90:459-66.

32. Bhawan J. MEL-5: a novel antibody for differential diagnosis of epidermal pigmented lesions of the skin in paraffin-embedded sections. Melanoma Res 1997;7:43-8.

33. Thomson TM, Mattes MJ, Roux L, et al. Pigmentation-associated glycoprotein of human melanomas and melanocytes: definition with mouse monoclonal antibody. J Invest Dermatol 1985;85:169-74.

34. Busam KJ, Chen YT, Old LJ, et al. Expression of Melan-A (MART-1) in benign melanocytic nevi and primary cutaneous melanoma. Am J Surg Pathol 1998;22:976-82.

35. Jungbluth AA, Busam KJ, Gerald WL, et al. A103: an anti-MelanA monoclonal antibody for the detection of malignant melanoma in paraffin-embedded tissue. Am J Surg Pathol 1998;22:595-602.

36. Chen YT, Stockert E, Jungbluth A, et al. Serological analysis of Melan-A (MART-1), a melanocyte specific protein homogeneously expressed in human melanomas. Proc Natl Acad Sci USA 1996;93:5915-9

37. Coulie PG, Brichard V, Van Pel A, et al. A new gene coding for a differentiation antigen recognized by autologous cytolytic $\mathrm{T}$ lymphocytes on HLA-A2 melanomas. J Exp Med 1994;180:35-42.

38. Saranton T, Chi DD, Garrison DA, et al. Melanoma-associated antigens as messenger RNA detection markers for melanoma. Cancer Res 1997;57:1371-6.

39. Fetsch PA, Cormier J, Hijazi YM. Immunocytochemical detection of MART-1 in fresh and paraffin-embedded malignant melanomas. J Immunother 1997;20:60-4.

40. de Vries TJ, Fowrkowr A, Wobbes T, et al. Heterogeneous expression of immunotherapy candidate proteins gp100, MART-1, and tyrosinase in human melanoma cell lines and in human melanocytic lesions. Cancer Res 1997;57:3223-9.

41. Blessing K, Sanders DSA, Grant JJH. Comparison of immunohistochemical staining of the novel antibody Melan-A with S-100 protein and HMB-45 in malignant melanoma and melanoma variants. Histopathology 1998;32:139-46.

42. Cormier JN, Hyazi YM, Abati A, et al. Heterogeneous expression of melanoma-associated antigens and HLA-A2 in metastatic melanoma in vivo. Int J Cancer 1998;75:517-24.

43. Wick MR. Immunohistologic features of melanocytic neoplasms. In: Dabbs DJ, editor. Diagnostic Immunohistochemistry. Philadelphia, PA: Churchill Livingstone; 2002. p. 147-62.

44. Griego RD, Zitelli JA. Mohs micrographic surgery using HMB-45 for a recurrent acral melanoma. Dermatol Surg 1998;24:1003-6.
45. Menaker GM, Chiang JK, Tabila B, Moy RL. Rapid HMB-45 staining in Mohs micrographic surgery for melanoma in situ and invasive melanoma. J Am Acad Dermatol 2001;44:833-6.

46. Zalla MJ, Lim KK, Dicaudo DJ, Gagnot MM. Mohs micrographic excision of melanoma using immunostains. Dermatol Surg 2000;26:771-84.

47. Bhardwaj SS, Tope WD, Lee PK. Mohs micrographic surgery for lentigo maligna and lentigo maligna melanoma using Mel-5 immunostaining: University of Minnesota experience. Dermatol Surg 2006;32:690-6.

48. Kelley LC, Starkus L. Immunohistochemical staining of lentigo maligna during Mohs micrographic surgery using MART-1. J Am Acad Dermatol 2002;46:78-84.

49. Albertini JG, Elston DM, Libow LF, et al. Mohs micrographic surgery for melanoma: a case series, a comparative study of immunostains, an informative case report, and a unique mapping technique. Dermatol Surg 2002;28:656-65.

50. Bricca GM, Brodland DG, Zitelli JA. Immunostaining melanoma frozen sections: the 1-hour protocol. Dermatol Surg 2004;30:403-8.

51. Kimyai-Asadi A, Ayala GB, Goldberg LH, et al. The 20-minute rapid MART-1 immunostain for malignant melanoma frozen sections. Dermatol Surg 2008;34:498-500.

52. Alessi E, Venegoni L, Fanoni D, Berti E. Cytokeratin profile in basal cell carcinoma. Am J Dermatopathol 2008;30:249-55.

53. Moll R, Moll I, Franke WW. Differences of expression of cytokeratin polypeptides in various epithelial skin tumors. Arch Dermatol Res 1984;276:349-63.

54. Perkins W, Campbell I, Leigh IM, MacKie RM. Keratin expression in normal skin and epidermal neoplasms demonstrated by a panel of monoclonal antibodies. J Cutan Pathol 1992;19:476-82.

55. Markey AC, Lane EB, Macdonald DM, Leigh IM. Keratin expression in basal cell carcinomas. Br J Dermatol 1992;126:15460.

56. Markey AC, Lane EB, Churchill LJ, et al. Expression of simple epithelial keratins 8 and 18 in epidermal neoplasia. J Invest Dermatol 1991;97:763-70.

57. Robinson JK, Gottschalk R. Immunofluorescent and immunoperoxidase staining of antibodies to fibrous keratin. Improved sensitivity for detecting epidermal cancer cells. Arch Dermatol 1984;120:199-203.

58. Robinson JK. Expression of keratin proteins in deeply invasive basal and squamous cell carcinoma: an immunohistochemical study. J Dermatol Surg Oncol 1987;13:283-94.

59. Setoyama M, Hashimoto K, Dinehart SM, et al. Immunohistochemical differentiation of basal cell epithelioma from cutaneous appendages using monoclonal anti-glycoprotein antibody TNKH1. Its application in Mohs' micrographic surgery. Cancer 1990;66:2533-40.

60. Tellechea O, Reis JP, Domingues JC, Baptista AP. Monoclonal antibody Ber EP4 distinguishes basal-cell carcinoma from squamous-cell carcinoma of the skin. Am J Dermatopathol 1993;15:452-5.

61. Kist D, Perkins W, Christ S, Zachary CB. Anti-human epithelial antigen (Ber-EP4) helps define basal cell carcinoma masked by inflammation. Dermatol Surg 1997;23:1067-70. 
62. Jimenez FJ, Burchette JL Jr, Grichnik JM, Hitchcock MG. Ber-EP4 immunoreactivity in normal skin and cutaneous neoplasms. Mod Pathol 1995;8:854-8.

63. Krunic AL, Garrod DR, Viehman GE, et al. The use of antidesmoglein stains in Mohs micrographic surgery. A potential aid for the differentiation of basal cell carcinoma from horizontal sections of the hair follicle and folliculocentric basaloid proliferation. Dermatol Surg 1997;23:463-8.

64. Leshin B, White WL. Folliculocentric basaloid proliferation. The bulge (der Wulst) revisited. Arch Dermatol 1990;126:900-6.

65. Krunic AL, Garrod DR, Madani S, et al. Immunohistochemical staining for desmogleins 1 and 2 in keratinocytic neoplasms with squamous phenotype: actinic keratosis, keratoacanthoma and squamous cell carcinoma of the skin. Br J Cancer 1998;77: 1275-9.

66. Smola MG, Soyer HP, Scharnagl E. Surgical treatment of dermatofibrosarcoma protuberans. A retrospective study of 20 cases with review of literature. Eur J Surg Oncol 1991;17:447-53.

67. Robinson JK. Dermatofibrosarcoma protuberans resected by Mohs' surgery (chemosurgery). A 5-year prospective study. J Am Acad Dermatol 1985;12:1093-8.

68. Jimenez FJ, Grichnik JM, Buchanan MD, Clark RE. Immunohistochemical margin control applied to Mohs micrographic surgical excision of dermatofibrosarcoma protuberans. J Dermatol Surg Oncol 1994;20:687-9.

69. Garcia C, Viehman G, Hitchcock M, Clark RE. Dermatofibrosarcoma protuberans treated with Mohs surgery. A case with CD34 immunostaining variability. Dermatol Surg 1996;22:177-9.
70. Smith SB, Farley MF, Albertini JG, Elston DM. Mohs micrographic surgery for granular cell tumor using S-100 immunostain. Dermatol Surg 2002;28:1076-8.

71. Albertini JG, Welsch MJ, Conger LA, et al. Infantile digital fibroma treated with Mohs micrographic surgery. Dermatol Surg 2002;28:959-61.

72. Marra DE, Schanbacher CF, Torres A. Mohs micrographic surgery of primary cutaneous mucinous carcinoma using immunohistochemistry for margin control. Dermatol Surg 2004;30:799-802.

73. Jimenez F, Clark RE, Buchanan MD, Kamino H. Lymphoepithelioma-like carcinoma of the skin treated with Mohs micrographic surgery in combination with immune staining for cytokeratins. J Am Acad Dermatol 1995;32(5 Pt 2):878-81.

74. Allee JE, Cotsarelis G, Solky B, Cook JL. Multiply recurrent trichilemmal carcinoma with perineural invasion and cytokeratin 17 positivity. Dermatol Surg 2003;29:886-9.

75. Hardaway CA, Graham BS, Barnette DJ, Feldman BD. Embryonal rhabdomyosarcoma presenting in an adult: a case report and discussion of immunohistochemical staining. Am J Dermatopathol 2003;25:45-52.

76. Robinson JK. Current histologic preparation methods for Mohs micrographic surgery. Dermatol Surg 2001;27:555-60.

Address correspondence and reprint requests to: Ayad E. Abrou, MD, Beacon Hill Dermatology, 2026N Beech Daly Road, Dearborn Heights, MI 48127, or e-mail: aabrou@yahoo.com 